\title{
Variabilidade circadiana da temperatura oral, timpânica e axilar em adultos hospitalizados*
}

\author{
CIRCADIAN VARIABILITY AT ORAL, TIMPANIC \\ AND AXILLARY TEMPERATURE IN HOSPITALIZED ADULTS
}

VARIABILIDAD CIRCADIANA DE LA TEMPERATURA TIMPÁNICA,
ORAL, Y DE LA AXILA EN ADULTOS HOSPITALIZADOS

Ana Leda Bertoncini Simões', Milva Maria Figueiredo De Martino²

\section{RESUMO}

Este estudo teve como objetivo verificar a variabilidade circadiana da temperatura timpânica, oral e axilar, considerando o ângulo de medida. Realizou-se em um hospital de ensino HC-Unicamp, na cidade de Campinas-SP. Os dados foram coletados a cada duas horas, por dois dias consecutivos, a partir do horário de vigília até as 22 horas, nas enfermarias de Cardiologia, Geral de Adultos e Gastroclínica. Os resultados mostraram que as medidas dos períodos matutino e vespertino, comparadas com as do período noturno, pela análise de variância, houve diferença significativa com $\mathrm{p}$ value $=0,0001$. O teste de Tukey confirmou a diferença no que se refere ao nível de significância $\mu$ $=0,05$. Identificou-se que as medidas do termômetro timpânico mostraram temperaturas mais elevadas em relação aos outros termômetros, confirmando os dados das literaturas internacionais e também demonstrando que a curva de ritmicidade circadiana foi similar à obtida pelas medidas do termômetro oral durante o período de vigília.

\section{DESCRITORES}

Temperatura corporal.

Ritmo circadiano.

Enfermagem.

\begin{abstract}
The aim of this study was to measure the circadian variability of patients' temperature in the eardrum, oral, and armpit positions considering the measurement angle. The study was carried out at the State University of Campinas' Clinics Hospital (HC Unicamp), a school hospital in the city of Campinas, State of São Paulo. Temperatures were taken in the Cardiology, Adult General Clinic and Gastric infirmaries every two hours in two consecutive days, from the time patients woke up until 10 PM. The results show a significant difference, $\mathrm{p}$-value $=0.0001$, between the morning and afternoon periods compared to the evening period. The Tukey test has also showed a difference. It was observed that the measurement in the eardrum position resulted in higher temperatures compared to the other positions, thus confirming data in the international literature. The circadian variability eardrum temperature was similar to the measurements of the oral temperature during the patients' awaked period.
\end{abstract}

\section{KEY WORDS}

Body temperature.

Circadian rhythm.

Nursing.

\section{RESUMEN}

El objetivo de este estudio fue verificar la variabilidad circadiana de la temperatura timpánica, oral y de la axila considerando el ángulo de medida. Se realizó en un hospital de enseñanza, elHC-Unicamp, Campinas. Los datos fueron colectados a cada dos horas, durante dos días consecutivos, a partir del horario de vigilia hasta las diez de la noche, en las enfermerías de Cardiología, General de Adultos y Gastroenterología . Los resultados demostraron que, comparando las medidas de los períodos matutino y vespertino, con las del período nocturno, hubo una diferencia significativa por el análisis de variancia, p-value 0,0001. El teste de Tukey confirmó la diferencia. Las medidas del termómetro timpánico mostraron temperaturas más elevadas en relación a los otros termómetros, lo que está de acuerdo con la literatura internacional. Se demostró que la curva de ritmo circardiano fue similar a las medidas del termómetro oral durante el período de vigilia.
* Extraído da dissertação "Estudo comparativo e variabilidade circadiana das temperaturas timpânica, oral e axilar em adultos hospitalizados",

Departamento de Énfermagem, Faculdade de Ciências Médicas, Universidade Estadual de Campinas, 2005.

1 Enfermeira. Mestre em Enfermagem pela Faculdade de Ciências Médicas, Universidade Estadual de Campinas, Campinas, SP, Brasil. albsimoes@ hotmail.com

2 Professora Associada do Departamento de Enfermagem, Faculdade de Ciências Médicas, Universidade Estadual de Campinas, Campinas, SP, Brasil. milva@unicamp.br

\section{DESCRIPTORES}

Temperatura corporal.

Ritmo circadiano.

Enfermería. 


\section{INTRODUÇÃO}

Mudanças fazem parte do cotidiano. Sem elas não há estímulos, crescimento ou desafios a serem vencidos. Melhorar a saúde de outrem é o principal desafio de um enfermeiro. $\mathrm{O}$ avanço tecnológico na área da saúde tem auxiliado de forma muito positiva esses profissionais; com novos equipamentos e novos conceitos é possível melhorar a cada dia a assistência de enfermagem prestada aos clientes.

A temperatura corporal começou a ser pesquisada por Sanctorius no ano de 1638. O estudioso foi o primeiro pesquisador a perceber as variações da temperatura. Então, a partir dessa percepção, começou a utilizar um instrumento para a verificação do calor corporal com o intuito de iniciar ou não uma terapêutica. A descoberta estimula diversos pesquisadores até os dias atuais ${ }^{(1)}$.

Em 1851, o pesquisador Wunderlich obteve um avanço importante para a medicina introduzindo um instrumento de temperatura, no qual originou o termômetro de mercúrio, utilizando a região axilar para a medida. Assim, definiu o estado da temperatura corporal no valor de $37^{\circ} \mathrm{C}$, com uma faixa de variabilidade entre $36,2^{\circ}$ e $37,5^{\circ} \mathrm{C}$. O valor da temperatura acima de $37,5^{\circ} \mathrm{C}$ definiuse como estado febril e maior ou igual a $38^{\circ} \mathrm{C}$ como estado de febre. Outro achado significativo foi a percepção do valor da temperatura em relação ao gênero, declarando que esta, em mulheres, poderia ser mais elevada quando comparada à temperatura em homens ${ }^{(1)}$.

Para tanto, a temperatura corporal passou a ser utilizada como um método de estimativa da temperatura central. O centro regulador da temperatura é o hipotálamo. Nele encontramos os termorreceptores que o auxiliam nessa função. A temperatura corporal de um indivíduo pode sofrer variações durante as 24 horas devido a vários fatores como: alterações emocionais, influência da temperatura ambiente, atividade física, roupas inadequadas, processos patológicos e ritmo circadiano. $\mathrm{O}$ monitoramento da medida da temperatura pode ser realizado por via oral, retal, axilar ${ }^{(2)}$ e pelo método timpânico.

Atualmente, ocorre a substituição gradual do termômetro de mercúrio por equipamentos eletrônicos digitais, mais rápidos, de fácil manuseio e mais seguros, pois o termômetro de vidro-mercúrio pode causar danos, tais como intoxicação devido à quebra do instrumento e em alguns casos lacerações de tecidos.

Surgiram novos instrumentos que incorporaram outros princípios para medida da temperatura como, por exemplo, o termômetro de radiação infravermelho e, dentre eles, os termômetros timpânicos. Fontes de inúmeras pesquisas, os termômetros timpânicos foram desenvolvidos para captar a temperatura do tímpano e tecidos vizinhos, local mais indicado para a medida da temperatura corporal.

\section{REVISÃO DA LITERATURA}

O hipotálamo é uma das principais glândulas do corpo humano, com peso de quatro gramas, representando menos de $1 \%$ da massa total do encéfalo. Situa-se na região basal do diencéfalo, próximo ao terceiro ventrículo, conhecido também como gânglio principal do sistema nervoso autônomo, constituído pela substância cinzenta que se agrupa em núcleos que são de difícil individualização ${ }^{(3)}$.

Por meio da estimulação do sistema endócrino, sistema motor somático e do sistema nervoso autônomo é possível exercer efeitos para manutenção de um ambiente interno relativamente constante no corpo - homeostasia. O hipotálamo é ainda o centro supra-segmentar mais importante do sistema nervoso autônomo, exercendo funções com outras áreas do cérebro e também com as do sistema límbico ${ }^{(4)}$.

\section{Variabilidade circadiana}

A Cronobiologia é considerada um ramo das Ciências Biológicas contemporâneas cujo objetivo é o estudo sistemático das características temporais da matéria viva em todos os seus níveis de organização e de ritmos biológicos como, por exemplo, as oscilações periódicas em variáveis biológicas e as mudanças associadas ao desenvolvimento ${ }^{(5)}$.

Existem dois mecanismos responsáveis pela organização temporal dos seres humanos: os fatores endógenos, ou relógio interno geneticamente programado da pessoa, que disparam um sistema composto de osciladores múltiplos, e os fatores exógenos, ou ocorrências ambientais horárias, que sincronizam os ritmos ${ }^{(6)}$.

Um dos pioneiros no campo da cronobiologia foi Franz Halberg, que instituiu o termo circadiano (circa significa quase, dies significa dia) para se referir aos ritmos que tenham uma duração de quase vinte e quatro horas. Podemos citar exemplos de ritmos circadianos que são conhecidos como o ciclo sono-vigília, repouso-atividade, claro-escuro e a temperatura corporal de um mamífero ${ }^{(7)}$.

Têm sido encontradas, numerosas variáveis fisiológicas e psicológicas demonstrando 24 horas de ritmo, incluindo a temperatura corpórea, o ciclo de sono, parâmetros cardiovasculares, performance cognitiva, fatores imunológicos e endócrinos, resposta terapêutica para certos tipos de medicação, além de variáveis de ansiedade e humor. Sob condições normais, atividades diárias e sono noturno, o sistema 
circadiano é sincronizado com as 24 horas do dia solar e pela alavanca externa à qual o relógio biológico é responsivo. O ritmo circadiano apresenta um componente endógeno, que reflete as respostas do desempenho interno, podendo trazer conseqüências ao trabalhador por turnos ${ }^{(8)}$.

Entretanto, os ritmos circadianos humanos são fortemente influenciados por fatores exógenos ou ambientais. Esses fatores são chamados de sincronizadores ou zeitgebers, neologismo alemão que significa doador de tempo $($ Zeit $=$ tempo; Gerber $=$ doador $)$. Para os seres humanos os zeitgerbers predominantes são as atividades sociais, como as refeições; estímulos auditivos, como os despertadores e outros estímulos ambientais que sugerem a hora do dia ou o dia da semana. Através dos quadros depressivos sazonais, foi observado que a luminosidade tem um papel importante como sincronizador de ritmos humanos ${ }^{(9-10)}$.

A influência do ritmo circadiano sobre a temperatura foi observada num estudo ${ }^{(11)}$ sobre a temperatura oral. O resultado observado foi a variação da temperatura oral no período de vigília, demonstrando valores de temperatura maiores no final da tarde e menores no início da manhã.

A variabilidade circadiana da temperatura oral, em enfermeiras do turno noturno, demonstrou que os valores das temperaturas oscilaram ao longo das 24 horas. Os valores das temperaturas foram elevados quando comparados com os valores dos sujeitos que trabalhavam durante o dia, indicando uma possível dessincronização dos ritmos biológicos ${ }^{(10)}$.

Pesquisa realizada sobre a técnica de medida para a temperatura timpânica, utilizando termômetro digital infravermelho teve como objetivo principal analisar as medidas de temperaturas timpânica e oral em sujeitos $(n=103)$ para detectar diferenças nos valores dessas medidas de acordo com sua angulação e locais de medidas. As medidas foram realizadas em clientes internados na unidade de cardiologia e ambulatório do Hospital de Clínicas da Unicamp. Os resultados mostraram que não houve diferença significativa entre as temperaturas timpânica, medidas em cada conduto auditivo, e a temperatura oral; no entanto, a temperatura timpânica medida em relação aos ângulos anterior e posterior de cada conduto auditivo apresentou diferença estatisticamente significativa $(\mathrm{p}=0,0001 \text { - Wilcoxon })^{(12)}$.

O conduto auditivo divide-se em três porções: o ouvido externo, o ouvido médio e ouvido interno. A membrana timpânica localiza-se no final do ouvido externo, separandoo do ouvido médio. Tem forma cônica, com área total de $85 \mathrm{~mm}^{2}$, coloração cinza pérola e é constituída por três camadas: a externa, de origem epitelial; a média, de maior importância, formada por um sistema de fibras colagênicas, de qualidade vibratória; e a interna, formada pela mucosa da caixa do tímpano $^{(13)}$. O responsável pela circulação do ouvido interno é a artéria labiríntica, conhecida também por artéria auditiva interna. Essa artéria penetra no ouvido interno juntamente com os pares de nervos cranianos VII e VIII, divide-se em artéria coclear e artéria vestibular, a qual irriga a maior parte do labirinto posterior ${ }^{(13)}$.

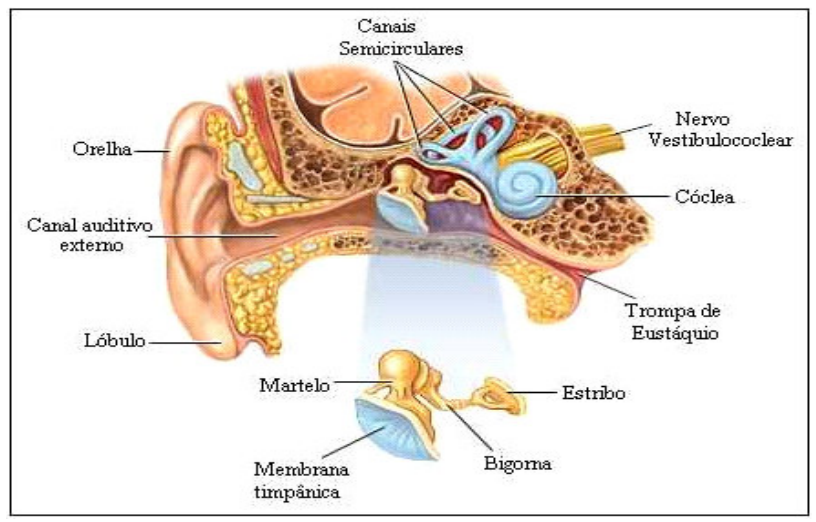

Figura 1 - Corte verticotransversal do ouvido direito ${ }^{(14)}$

\section{Temperatura timpânica}

O princípio de funcionamento do termômetro timpânico baseia-se na medida da quantidade de energia infravermelha emitida pela membrana timpânica e de tecidos vizinhos, que converte o fluxo de calor em corrente elétrica. A corrente de voltagem converte seu alimento em uma voltagem de produção dentro de um Multiplexer e depois realiza a conversão analógico-digital, demonstrando em seu visor o valor da temperatura corporal verificada ${ }^{(15)}$.

Alguns autores elucidaram as vantagens e desvantagens sobre os locais de medidas de temperatura corporal utilizadas no atendimento de emergência pediátrica. Temperatura oral, vantagens: facilidade de aplicação, método não invasivo. Desvantagens: dificuldade na utilização em crianças jovens, o uso de máscara de oxigênio ou tubo oro traqueal, contra-indicada em crianças com traumas maxilofacial e incerteza com clientes com hipotermia ${ }^{(16)}$.

Axilar, vantagens: facilidade de uso, método não invasivo. Desvantagens: não reflete a temperatura central, também questionável exatidão em clientes com hipotermia, facilidade do deslocamento durante a medida e ampla variabilidade ${ }^{(16)}$.

Membrana timpânica, vantagens: método não invasivo, rapidez, facilidade de uso, proximidade com o hipotálamo, indica hipotermia, exatidão de medida com ou sem otite média. Desvantagens: técnica inadequada pode afetar a medida, contra-indicada em clientes com fratura maxilofacial, base de crânio e otorragia; pode sofrer influência da temperatura ambiente; cerúmen pode apresentar falsa medida; imobilização cervical dificulta a medida ${ }^{(16)}$.

Retal, vantagens: facilidade na introdução e na medida da temperatura visceral e pode ser um índice favorável da temperatura central. Desvantagens: causa desconforto físico e emocional; presença de fezes no reto afeta a exatidão da medida; possibilidade de hemorragia e contra-indicado em clientes com trauma retal ou alguma doença local ${ }^{(16)}$. 
Um estudo com 89 crianças com e sem o diagnóstico de otite média com secreção mostraram que as que apresentavam otite com secreção foram submetidas a procedimento cirúrgico eletivo para inserção de um anel metálico e avaliação se essa secreção afetava a medida da temperatura tim-pânica. Como método de comparação, utilizou o termômetro timpânico aplicando em ambos condutos auditivos e o termômetro digital na região axilar. A análise estatística mostrou que não houve diferença significativa entre as medidas do termômetro timpânico em ambos condutos, com ou sem a presença de secreção. No entanto, os autores apontam a existência de algumas variáveis, como a idade e o gênero, as quais podem interferir nas temperaturas, por exemplo: a mulher durante seu período ovulatório tem a temperatura elevada e a criança normalmente apresenta temperatura mais alta em relação aos adultos ${ }^{(17)}$. Essas variações foram relatadas em outros trabalhos ${ }^{(18-19)}$.

\section{OBJETIVO}

Considerando-se os aspectos relacionados, este estudo teve como objetivo:

- Verificar a variabilidade circadiana da temperatura timpânica, oral e axilar considerando o ângulo de medida.

\section{MÉTODO}

A pesquisa caracterizou-se como um estudo descritivo e observacional. Inicialmente o projeto desta pesquisa foi encaminhado ao Comitê de Ética e Pesquisa da Faculdade de Ciências Médicas-Unicamp, tendo sido aprovado. A pesquisa desenvolveu-se em um hospital de ensino, Hospital de Clínicas da Universidade Estadual de Campinas (HCUnicamp) situado na cidade de Campinas, São Paulo. Foram selecionadas para a coleta as enfermarias de Cardiologia, Gastroclínica e a Enfermaria Geral de Adultos.

Os instrumentos realizados para a coleta de dados foram: planilha elaborada no software Excel 2000; termômetro infravermelho marca Omron - modelo MC505; termômetro digital marca More Fitness - modelo MF102; termômetro de vidro mercúrio padrão internacional, para a verificação da temperatura ambiente. Os termômetros foram calibrados no laboratório da Faculdade de Engenharia Mecânica (FEM)Unicamp, conforme seus princípios de funcionamento.

A população alvo deste estudo constituiu-se de 15 clientes, computando 18 medidas de temperatura de cada termômetro, durante o mês de fevereiro de 2004, sendo eles do sexo masculino com faixa etária entre 22 e 75 anos, que aceitaram participar voluntariamente da pesquisa e assinaram o termo de consentimento livre e esclarecido. Os aspectos éticos foram cumpridos a partir dos princípios enunciados na Declaração de Helsinque III, 2001 e nos termos da Resolução 196 de 10 de outubro de 1996.
Foram excluídos clientes menores de 18 anos; que fizeram uso de antitérmico no período de duas horas antes da coleta e, durante a coleta, clientes que apresentaram febre. Para a temperatura oral foram excluídos aqueles que apresentaram: taquipnéia, sinal de infecção e lesões na cavidade oral. Para a temperatura axilar, lesões nas axilas. Para a verificação da temperatura timpânica foram considerados: acúmulo de cerúmen e otite média.

Os responsáveis pela coleta de dados foram dois técnicos de enfermagem da Unidade de Emergência Referenciada (UER) HC - Unicamp e a autora do trabalho. Foi aplicado treinamento prévio para o manuseio dos termômetros infravermelho e digital e para a execução correta das técnicas de verificação das temperaturas.

Antes de iniciar a coleta também foi verificada a temperatura ambiente de cada enfermaria nos seguintes períodos: manhã às $5 \mathrm{~h} 30 \mathrm{~min}$, tarde às $14 \mathrm{~h}$ e noite às $20 \mathrm{~h}$.

A coleta dos dados foi efetuada a cada duas horas, por dois dias consecutivos a partir do horário de vigília do cliente até às vinte e duas horas, com a finalidade de verificar a variabilidade circadiana de todas as medidas. A higienização dos termômetros foi realizada antes e após cada procedimento com uso de algodão embebido em álcool a 70\%.

As realizações das medidas das temperaturas corporais foram feitas de acordo com as técnicas descritas nos estudos para os termômetros timpânicos ${ }^{(12,15,20-21)}$, para termômetro oral ${ }^{(22)}$ e para termômetro axilar ${ }^{(23)}$.

\section{Técnica utilizada para a verificação da temperatura oral(22)}

- acomodar o paciente em decúbito dorsal;

- instruir o paciente sobre o que será feito, orientando-o para manter sua boca aberta até que o termômetro esteja em posição adequada;

- introduzí-lo sob a língua e deslizá-lo lentamente ao longo da linha da gengiva, em direção à porção posterior da boca;

- despender de 4 a 5 segundos para atingir o espaço sublingual posterior $\mathrm{D}$;

- solicitar ao paciente o fechamento da forma acomodada de seus lábios ao redor do termômetro;

- fazer a leitura e removê-lo;

- anotar o resultado em folha indicada.

\section{Técnica utilizada para a verificação da temperatura axilar ${ }^{(23)}$}

- colocar o paciente em decúbito dorsal;

- explicar o cuidado que lhe será prestado e orientá-lo quanto ao movimento de abdução e adução do braço, fazer abdução do braço $\mathrm{D}$ até um ângulo de $35^{\circ} \mathrm{C}$;

- afastar a roupa do paciente para expor totalmente a axila;
Variabilidade circadiana da temperatura oral, timpânica e axilar em adultos hospitalizados Simões ALB, De Martino MMF 
- secar com lençol a axila do paciente, realizar movimentos de absorção;

- pegar o termômetro e posicioná-lo paralelamente à parede medial da axila do paciente;

- encostar sua extremidade no ápice da axila;

- girá-lo, posicionando-o perpendicularmente à parede medial;

- solicitar ao paciente que faça adução do braço para fechar a cavidade axilar;

- flexionar o antebraço e apoiá-lo sobre o tórax;

- soltar e marcar o tempo;

- aguardar 20 segundos;

- fazer a leitura;

- pedir ao paciente para estender o antebraço e fazer abdução do braço;

- retirar o termômetro;

- anotar o resultado em folha indicada.

O procedimento detalhado para a obtenção da leitura de temperatura timpânica (T.T.), é descrito a seguir ${ }^{(12)}$ :

- Acomodar o paciente em decúbito dorsal ou sentado;

- Explicar o procedimento e orientá-lo quanto à rotação da cabeça a $20^{\circ} \mathrm{C}$ do local de escolha para a verificação da temperatura;

- Avaliar o conduto auditivo e observar a presença de cerúmen;

- Tracionar gentilmente a orelha para cima e para trás, até o final da tomada da temperatura;

- Introduzir o termômetro no conduto auditivo delicadamente;

- Acoplá-lo bem no conduto;

- Ligar o termômetro e aguardar o sinal sonoro.

Este procedimento foi realizado para o termômetro ajustado na posição anterior, temperatura timpânica anterior (T.T.A.), embasado no estudo das autoras ${ }^{(13)} \mathrm{o}$ conduto auditivo escolhido aleatoriamente foi o esquerdo.

Como critérios de febre nesse estudo, adotaram-se os seguintes valores verificados pela temperatura oral $37,8^{\circ} \mathrm{C}$ e $38,3^{\circ} \mathrm{C}^{(24)}$.

Os resultados obtidos no experimento foram estabelecidos conforme análise descritiva para calcular a média, técnica paramétrica de análise de variância e teste de Tukey para comparações múltiplas. O nível de significância adotado foi $\mu=0,05$. Para considerar diferenças estatisticamente significante, foi adotado p-value $<0,05$. As análises foram realizadas usando o software estatístico (Statistical Analysis System - SAS) versão 6.12.

\section{RESULTADOS E DISCUSSÃO}

Nesta pesquisa tivemos o propósito de analisar a variabilidade circadiana da temperatura timpânica, oral e axilar, considerando o ângulo de posicionamento, e compará-las entre si.

Na Figura 2 pode-se observar uma curva de variabilidade circadiana na qual a primeira medida da temperatura timpânica apresentou seu valor em torno de $37,0^{\circ} \mathrm{C}$. Verificase também que a temperatura timpânica manteve-se constante nos períodos matutino e vespertino, elevando-se a partir das $18 \mathrm{~h}$ atingindo seu pico de $38,4^{\circ} \mathrm{C}$, às $20 \mathrm{~h}$. A temperatura oral apresentou sua primeira leitura em $36,5^{\circ} \mathrm{C}$, mantendo-se constante até o final do período vespertino, atingindo também seu pico às $20 \mathrm{~h}$. A leitura da temperatura axilar teve inicio em $36,2^{\circ} \mathrm{C}$ e observou-se que ao longo dos períodos matutino e início do vespertino manteve-se também constante, iniciando sua elevação por volta das $16 \mathrm{~h}$, atingindo o pico arrastado às $22 \mathrm{~h}$. Os valores obtidos demonstraram a presença de ritmicidade circadiana ${ }^{(11)}$.

Nota-se que ao longo dos horários de coleta a temperatura timpânica permaneceu com valores mais elevados quando comparada às outras temperaturas. As medidas realizadas na membrana timpânica justificam os valores elevados devido à proximidade com o hipotálamo, centro regulador da temperatura.

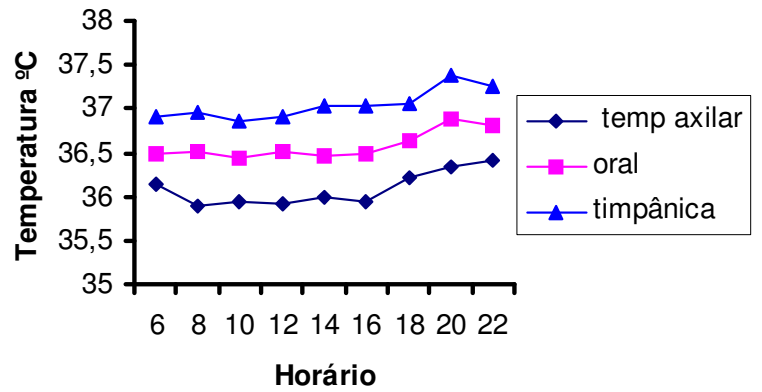

Figura 2 - Valores médios das temperaturas oral, axilar e timpânica $(n=30)$, Campinas - 2004

Após a análise dos valores médios das temperaturas axilar, oral e timpânica foi efetuado um estudo individual entre as temperaturas desses clientes saudáveis para avaliar a variabilidade circadiana de cada indivíduo, conforme Figura 3.

A Figura 3 ilustra dados do cliente 2, com 26 anos.

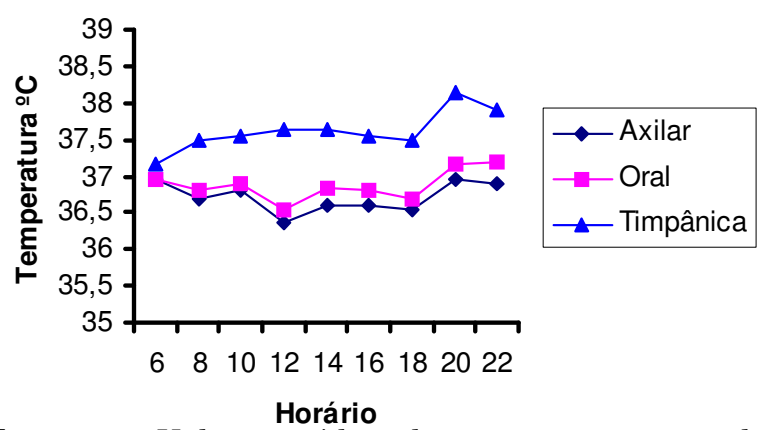

Figura 3 - Valores médios das temperaturas, axilar, oral e timpânica, paciente 2, Campinas - 2004 
A temperatura timpânica iniciou seu ciclo em $37,1^{\circ} \mathrm{C}$, elevando-se durante o período matutino e vespertino e mantendo sua característica inerente, atingindo o pico máximo às $20 \mathrm{~h}$, conforme o padrão cronobiológico, com a temperatura elevando-se no final da tarde.

As temperaturas oral e axilar apresentaram as mesmas variações. A oral obteve temperatura pouco mais elevada quando comparada à axilar. Ambas mantiveram a curva de ritmicidade circadiana apresentando oscilações no período de vigília como ilustra na Figura 3.

Tabela 1 - Distribuição da média geral e desvio-padrão da variável temperatura oral, axilar e timpânica em pacientes hospitalizados, Campinas - 2004

\begin{tabular}{ccccc}
\hline $\begin{array}{c}\text { Temperatura } \\
\text { Geral }\end{array}$ & Média & DP & Mínimo & Máximo \\
\hline$(\mathrm{N}=810)$ & $36,57^{\circ} \mathrm{C}$ & $0,73^{\circ} \mathrm{C}$ & $33,1^{\circ} \mathrm{C}$ & $38,5^{\circ} \mathrm{C}$
\end{tabular}

* $\mathrm{p}=0,05 ; \mathrm{dp}=$ desvio-padrão; análise de variância

Comparando-se as médias gerais das temperaturas dos termômetros sem considerar os horários de medidas, a análise de variância mostrou que houve diferença significativa entre os valores da temperatura $p$-value $=0,0001<\propto$ $=0,05$. O teste de Tukey mostrou que houve diferença significativa entre as médias das temperaturas dos termômetros timpâ-nico $\left(37,3^{\circ} \mathrm{C}\right)$, oral $\left(36,8^{\circ} \mathrm{C}\right)$ e o axilar $\left(36,3^{\circ} \mathrm{C}\right)$, com significância $\propto=0,05$.

Avaliando as médias dos horários medidos pelos termômetros no período noturno, observou-se que não houve diferença significativa entre os horários medidos no período noturno com p-value $=0,8>\propto=0,05$. O teste de Tukey mostrou que não houve diferença significativa entre as médias dos horários das temperaturas no período noturno, ou seja, às $20 \mathrm{~h}$ e às $22 \mathrm{~h}$ foram $36,8^{\circ} \mathrm{C}$ ao nível de significância de $\propto=0,05$.

A utilização da técnica correta do termômetro timpânico evita erro sistemático em suas medidas. Os estudos ${ }^{(25)}$ mostraram a importância do uso da mão de maior destreza na coleta das temperaturas, pois através dela realiza-se a retração correta do ouvido, diminuindo a probabilidade de erro de leitura no canal auditivo, o que pode apresentar resultado com valor falsamente baixo devido à dispersão do fluxo de calor dentro do conduto auditivo. Este estudo confirmou os achados citados acima, em que algumas leituras apresentaram valores baixos devido ao mau posicionamento no canal.

Pesquisas realizadas compararam as temperaturas axilares e timpânicas em ambos os ouvidos, em crianças com diagnóstico de otite média ${ }^{(17)}$. Utilizando-se do teste pareado, demonstraram que a temperatura timpânica bilateral e axilar não foram estatisticamente diferentes ao nível de significância $\propto=0,05$. Portanto, observou diferença significativa entre os condutos direito e esquerdo. Este estudo mostrou diferença significativa entre a temperatura timpânica e axilar demonstrada no teste análise de variância realizado para as médias entre os termômetros, $\mathrm{p}$-value $=0,0001<\propto=0,05$. Pelo teste de Tukey confirmou-se a diferença estatisticamente significativa entre as médias dos termômetros, o termômetro timpânico anterior (TTA) > termômetro oral (TO) > termômetro axilar (TAX).

Numa pesquisa recente analisando as medidas de temperaturas timpânica e oral em sujeitos $(n=103)$ para detectar diferenças nos valores dessas medidas de acordo com sua angulação e locais de medidas, realizadas em clientes internados na unidade de cardiologia e ambulatório do Hospital de Clínicas (HC) da Unicamp. Os resultados mostraram que não houve diferença significativa entre as temperaturas timpânica, medidas em cada conduto auditivo, e a temperatura oral. No entanto, a temperatura timpânica medida em relação aos ângulos anterior e posterior de cada conduto auditivo apresentou diferença estatisticamente significativa ( $\mathrm{p}=0,0001$ - Wilcoxon). Assim, este estudo realizou as leituras utilizando a angulação na posição anterior devido ao melhor ajuste no conduto confirmando o achado citado ${ }^{(12)}$.

\section{CONCLUSÕES}

Os dados obtidos neste estudo identificaram que as medidas realizadas com o termômetro timpânico apresentaram temperatura mais elevadas em relação aos outros tipos de termômetros utilizados (oral e axilar), confirmando literaturas internacionais.

A utilização do ângulo correto para a temperatura timpânica deve ser respeitada conforme trabalhos citados no artigo, pois o ângulo anterior para leitura oferece um encaixe correto minimizando erros de leituras. Segundo pesquisas ${ }^{(25)}$, a mão de maior destreza contribui para uma técnica correta de leitura.

Verificou-se que o emprego do termômetro timpânico para estudo da ritmicidade circadiana foi similar à curva encontrada pela medida do termômetro oral durante o período de vigília. O termômetro timpânico demonstrou compatibilidade de leitura realizada em outras pesquisas. Também oferecendo mais uma opção de medida, o conduto auditivo tem proximidade com o centro controlador da temperatura: o hipotálamo.

O uso do termômetro timpânico apresentou vantagens como rapidez de leituras identificando a temperatura em 2 segundos, técnica não invasiva, indolor, que não entra em contato com a membrana timpânica e apresenta facilidade de uso. Logo, sugere-se que equipes de enfermagem ampliem o conhecimento do termômetro timpânico - o qual será de extrema valia principalmente quanto ao tempo de medida - nas unidades de atendimento de emergências e clínicas pediátricas.

Preconiza-se também um treinamento breve para demonstrar a técnica adequada para enfermagem evitando erros sistemáticos de leitura. 
Quanto ao termômetro axilar, observa-se a necessidade de secar as axilas para evitar valores incorretos. $\mathrm{O}$ uso em clientes com síndrome comsuptiva, emagrecidos, desnutridos o termômetro não se encaixa corretamente nas axilas devido a depressão do local, dificultando a leitura e permitindo registrar falsas temperaturas.

\section{REFERÊNCIAS}

1. Sund-Levander M, Forsberg C, Wahren LK. Normal oral, rectal, tympanic and axillary body temperature in adult men and women: a sistematic literature review. Scand J Caring Sci. 2002;16(2):122-28.

2. Yaron M, Lowenstein SR, Mclain JK. Measuring the accuracy of the infrared tympanic thermometer: correlation does not signify agreement. J Emerg Med. 1995;13(5):671-21.

3. Machado ABM. Neuroanatomia funcional. $2^{\mathrm{a}}$ ed. São Paulo: Atheneu; 1998. Estrutura e função do hipotálamo; p. 229-36.

4. Noback CR, Strominger NL, Demarest RJ. Neuroanatomia: estrutura e função do sistema nervoso humano. $5^{\text {a }}$ ed. São Paulo: Premier; 1999. Hipotálamo; p. 295-309.

5. Cippola-Neto J, Marques N, Menna Barreto LS, editores. Introdução ao estudo da cronobiologia. São Paulo: Ícone; 1988.

6. Monk TH. The relationship of chronobiology to sleep schedules and performance demands. Work Stress. 1990;4(3):227-36.

7. Halberg F, Caradente F, Cornélissen G, Katinas GS. "Glossary of chronobiology”. Cronobiologia. 1977;4 Supl 1:1-189.

8. Scott A.J. Shift work and health. Prim Care. 2000;27(4):1057-78.

9. Aschoff J. Exogeneous and endogeneous components in circadian rhythms. Cold Spring Harbor Symp Quant Biol. 1960;25(1):11-28.

10. DeMartino MMF. Estudo da variabilidade circadiana da temperatura oral, do ciclo vigília-sono e de testes psicofisiológicos de enfermeiras em diferentes turnos de trabalho [tese]. Campinas: Instituto de Biologia, Universidade Estadual de Campinas; 1996

11. Afeche SC. Conceitos fundamentais da ritmicidade biológica. In: Marques N, Menna- Barreto L, organizadores. Cronobio-logia: princípios e aplicações. São Paulo: EDUSP; 1997. p. 34-50.

12. DeMartino MMF, Simões ALB. A comparative study of tympanic and oral temperatures in healthy adults. Rev Ciênc Med. 2003;12(2):115-21.

13. Hungria H. Otorrinolaringologia. $7^{a}$ ed. Rio de Janeiro: Guanabara Koogan; 1995.

14. United States National Library Of Medicine. Medical Encyclopedia-Ear Anatomy [encyclopedia on the Internet]. [cited 2003 Out 12]. Available from: http://www.nlm.nih.gov/ medlineplus/ency/encyclopedia_E-Ep.htm
Existem ainda estudos internacionais que demonstram dúvidas sobre a utilização do emprego do termômetro timpânico. Em nosso meio foram mencionadas duas situações apresentando e não apresentando diferenças estatisticamente significativa em seu uso. Portanto, novos estudos deverão ser realizados com um número maior de sujeitos, contribuindo com os achados descritos nesta pesquisa.

15. Betta V, Cascetta F, Sepe D. An assessment of infrared tympanic thermometers for body temperature measurement. Physiol Meas. 1997;18(3):215-25.

16. Bernardo LM, Henker R, O'Connor J. Temperature measurement in pediatric trauma patients: a comparison of thermometry and measurement routes. J Emerg Nurs. 1999;25(4):327-9.

17. Robb PJ, Shahab R. Infrared transtympanic temperature measurement and otitis media with effusion. Int J Pediatr Otorhinolaryngol. 2001;59(3):195-200.

18. Chamberlain JM, Terndrup TE, Alexander DT, Silverstone FA, Wolf-Klein G, O'donnell R. Determination of normal ear temperature with an infrared emission detection thermometer. Ann Emerg Med. 1995;25(1):15-20.

19. Chaud MN, Peterlini MAS, Harada MJCS, Pereira SR. O cotidiano da prática de enfermagem pediátrica. São Paulo: Atheneu; 1999. p. 89-91.

20. Greenleaf JE, Castle BL. External auditory canal temperature as an estimate of core temperature. J Appl Physiol.1972; 32(2):194-8

21. Doyle F, Zehner WJ, Terndrup TE. The effect of ambient temperature extremes on tympanic and oral temperatures. Am J Emerg Med. 1992;10(4):285-9.

22. Mamede MV, Carvalho EC, Cunha AMP. Técnicas de enfermagem. São Paulo: Savier; 1981. p. 50-63.

23. Angerami ELS. Estudo da temperatura corporal, medida na axila para estabelecer limites da classificação do normal, em colegiais do sexo feminino. Rev Bras Enferm. 1971; 24(1/2): 17-26.

24. Baldy JLS. Diagnóstico clínico síndrome infecciosa. In: Amato Neto V, Baldy JLS. Doenças transmissíveis. $3^{\mathrm{a}}$ ed. São Paulo: Sarvier; 1991.p. 91-9.

25. Terndrup TE, Rajk J. Impact of operator technique and device on infrared emission detection tympanic thermometry. J Emerg Med. 1992;10(6):683-87. 